\title{
Development of Multichannel Interdigitated Capacitor Based Sourness Sensor
}

\author{
Md. Rajibur Rahaman Khan, Hyun-Min Jeong, Sae-Wan Kim, Jin-Beom Kwon, and Shin-Won Kang* \\ School of Electronics Engineering, Kyungpook National University, \\ 80 Daehakro, Bukgu, Daegu 41566, South Korea. \\ *swkang@knu.ac.kr
}

\begin{abstract}
:
In our study, we designed and developed a multichannel interdigitated capacitor (IDC) based sourness sensor, that is based on the principle of capacitance variation. Four different types of solvatochromic dyes namely, Nile red, Reichardt's dye (R-dye), auramine-O, and rhodamine-B were individually mixed with polyvinylchloride (PVC) polymer and dimethylacetamide (DMAC) solution to make four different types of sourness sensitive sensing solutions. Thoes solutions were individually inserted into four interdigitated electrodes by a spin coater and dry at the room temperature to get four IDC sourness sensors of the array. In our study, when the IDC sensor comes in to contact with any sour-solution then the dielectric constant of the sensing membrane change, which in turn change the capacitance of IDC sensor. The sensitivity of the sensor was approximately $3 \mathrm{nF} /$ decade. The proposed multichannel IDC sensor can detect sourness of any sour-solution from $1 \mu \mathrm{M}$ to $1 \mathrm{M}$ with fast response and recovery time. The response and recovery time of the proposed sensor was approximately $20 \mathrm{~s}$ and $23 \mathrm{~s}$, respectively. We compared the sensing performance of the designed multichannel IDC sensor with a potentiometric sensor with respect to dynamic range width, and response time, we found that the proposed IDC sensor has better sensing performance.
\end{abstract}

Key words: Interdigitated capacitor, solvatochromic dye, sourness-sensor, dielectric constant, response time

\section{Experimental Results}

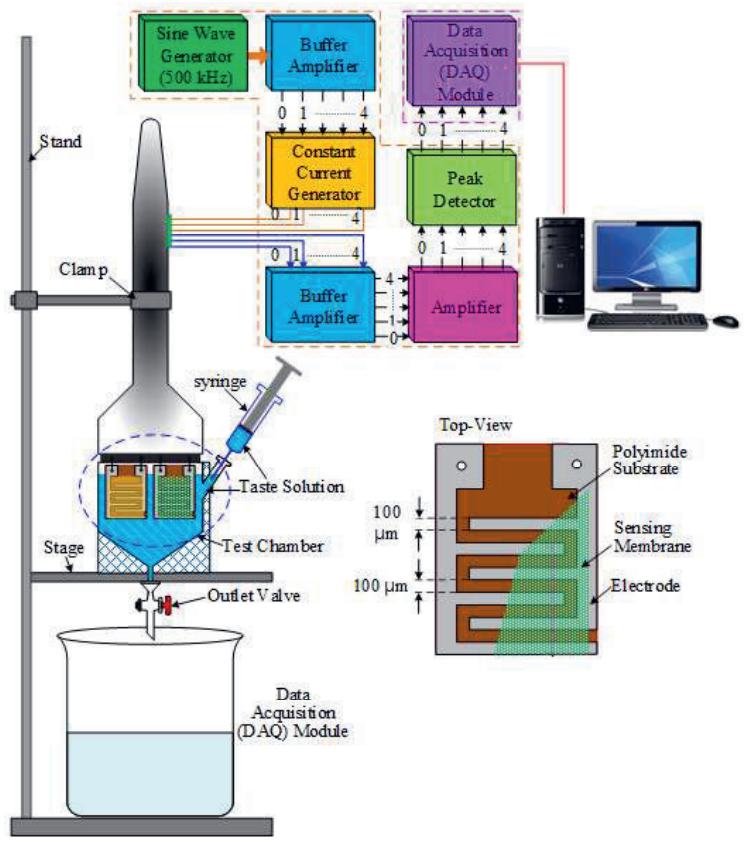

Fig. 1. Schematic diagram of the proposed multichannel sourness sensor.

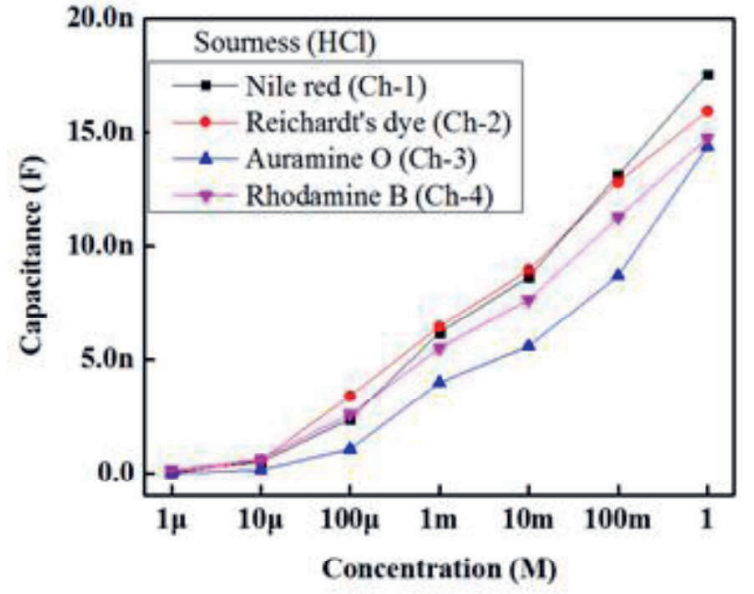

Fig.2. Sensing response of the designed solvatochromic containing multichannel IDC sensor.

Acknowledgments: This study was supported by the BK21 Plus project funded by the Ministry of Education, Korea (21A20131600011) and the National Research Foundation of Korea (NRF) grant funded by the Korea Government (MSIP) (No. NRF 2017R1D1A3B03032042). 